\title{
Análisis fitoquímico de hojas de Cordia stellifera utilizada como antídoto contra mordedura de serpiente en la comunidad de Lancetilla
}

Karen Castro, Inés Castro, Gabriela Flores, Jeimy Mejía, Bessy Rodríguez ${ }^{1}$ Ana Carolina Arévalo ${ }^{2}$

\section{RESUMEN}

Mediante un sondeo etnobotánico realizado en la comunidad de Lancetilla, se identificó que la especie Cordia stellifera, conocida comúnmente como sombra de ternero, es utilizada por la población de la comunidad para tratar los efectos de mordeduras de serpiente. Se administra por vía tópica, macerando las hojas y aplicando el macerado directamente sobre la zona afectada en forma de cataplasma.

A través de este estudio fitoquímico se pretende identificar la presencia/ausencia de los principales núcleos de metabolitos secundarios y de esta forma aportar nuevos datos químicos que avalen el uso antiofídico reportado por la comunidad, tomando en consideración que las estadísticas nacionales indican que en el departamento de Atlántida se reporta anualmente un elevado número de casos por mordeduras de serpiente, por lo que se considera importante el estudio fitoquímico de esta especie.

En el presente trabajo se demuestra la presencia de flavonoides, cumarinas, taninos y heterosidos cianogenéticos en las hojas de Cordia stellifera, de los cuales se tienen reportes previos acerca de que algunos de estos presentan actividad antiofídica, lo que podría justificar el uso etnobotánico que le da la comunidad a esta planta.

Palabras clave: Cordia stellifera, mordeduras de serpiente, análisis fitoquímico.

\section{ABSTRACT}

By means of an etnobotanical study, realized in the community of Lancetilla, the species Cordia stellifera, commonly known as "Sombra de ternero" (calf shadow)

\footnotetext{
${ }^{1}$ Estudiantes de la clase Farmacognosia I, Departamento de Control Químico, Facultad de Química y Farmacia, UNAH.

${ }^{2}$ Asesora, Departamento de Control Químico, Facultad de Química y Farmacia, UNAH. Contacto: ana.arevalo@unah.edu.hn
} 
was identified as being used by the population of the community to treat the effects of snakebite, by topical use, forming a paste from the leaves and applying it directly over the affected area in the form of a plaster.

The aim of this phytochemical study is to identify the presence/absence of the principal secondary metabolites and in this way, to provide new chemical data that will demonstrate the antiofidic use reported by the community, taking into consideration that national statistics indicate that the department of Atlantida annually reports an elevated number of cases of snakebite. The present study indicated the presence of flavonoids, coumarins, tannins and cianogenetic glycosides in the leaves of Cordia stellifera. There are previous reports that some flavonoids, coumarins and tannins present antiofidic activity which could justify the etnobotanic use that the community gives to this plan.

Key words: Cordia stellifera, snakebite, phytochemical analysis.

\section{INTRODUCCIÓN}

Los productos naturales han servido como fuente de medicamentos desde la antigüedad, por eso más de la mitad de los principios activos usados en farmacia actualmente son derivados de productos naturales (Newman y Cragg, 2012). En consecuencia, cada día se presta más atención al estudio de las plantas medicinales, de forma que la etnobotánica, la fitoterapia y la fitoquímica están tomando un auge insospechado, tanto en la práctica de la medicina complementaria como en el ámbito académico (Beyra, León e Iglesias, 2004).

El $80 \%$ de la población mundial, más de cuatro mil millones de personas, utilizan las plantas como principal remedio medicinal, según señala la OMS (Akerele, 1993). Esta práctica está asociada al empirismo en muchos casos, ya que faltan estudios químicos, clínicos y epidemiológicos que confirmen de forma fehaciente los efectos terapéuticos de las plantas y los principios activos responsables. Precisamente, hoy en día es en los países del tercer mundo en donde la medicina tradicional sobrevive de una forma más auténtica, lo cual hace más fácil identificar las plantas que necesitan ser científicamente evaluadas. Al parecer, la distancia entre la medicina tradicional y la ortodoxa empieza a acortarse y que ya no se considera la primera como un obstáculo para el progreso científico .

Honduras presenta variadas condiciones climáticas y geográficas, lo que ha permitido el desarrollo de una gran variedad de especies medicinales, alimenticias y ornamentales que constituyen una enorme riqueza para el país (Nelson,1986); 
además, se cuenta con especies medicinales que han sido utilizadas desde tiempos inmemoriales, pero que hasta el momento se desconoce su composición química, por lo que es necesario realizar investigaciones fitoquímicas sobre la medicina tradicional para aprovechar estos recursos con una base científica sólida.

Con el objetivo de orientar la investigación fitoquímica hacia aquellas plantas que cuentan con un aval tradicional, se realizó un sondeo etnobotánico en la comunidad de Lancetilla, municipio de Tela, en el departamento de Atlántida, de una de las especies de uso tradicional identificadas en la zona: la Cordia stellifera boraginaceae, conocida comúnmente como sombra de ternero. La población utiliza las hojas de este árbol para contrarrestar el veneno de la mordedura de serpiente, macerando las hojas y utilizándolas en forma de cataplasma que se aplicada directamente en la zona de la mordedura.

Resulta oportuno el estudio de esta especie ya que las mordeduras de serpientes representan un problema de salud pública; así, en el año 2012, en el país se reportaron 641 casos de mordeduras de serpientes; en el 2013, hasta la semana 42 se registraron 575 casos, de los cuales el $14 \%$ sucedió en el departamento de Atlántida(Solórzano, 2013).

Se ha estimado que cada año se reportan cinco millones de picaduras de serpientes venenosas en humanos, especialmente en las zonas rurales de países subdesarrollados con escasa o nula capacidad hospitalaria o farmacológica; ante esta situación, la medicina tradicional basada en los vegetales se ha utilizado históricamente para el tratamiento de tales mordeduras . Sin embargo, la mayoría de los taxones que etnobotánicamente se registran con actividad antiofídica no han sido estudiados químicamente y no se conocen los principios activos responsables de dicha actividad.

Al respecto, en la literatura sobre este tema no existen estudios fitoquímicos de Cordia stellifera, por lo que con este trabajo de investigación se pretende determinar la presencia o ausencia de los metabolitos secundarios que podrían ser los responsables del efecto terapéutico manifestado por la comunidad para esta especie.

Cordia es un género de árboles y arbustos de la familia boraguinaceae. Se han identificado alrededor de 250 especies distribuidas en regiones cálidas del planeta . Los frutos, hojas, corteza, semillas y raíces de la mayoría de las especies del género Cordia han sido usadas en la medicina tradicional como cicatrizante, astringente, antiinflamatorio, antihelmíntico, antimalárico, diurético, supresor del apetito, supresor de la tos, infecciones urinarias y lepra (Thirupathi, 2008). 
Las plantas del género Cordia constituyen una fuente rica de fitoconstituyentes y observan un amplio rango de compuestos como flavonoides, alcaloides, cardioquinonas, fenilpropainoides, taninos y triterpenos; metabolitos secundarios a los que se les ha reportado un amplio rango de bioactividad. Para efectos del presente estudio se evaluó la presencia/ausencia de alcaloides, antraquinonas, flavonoides, taninos, cumarinas, heterósidos cardiotónicos y heterósidos cianogenéticos y se demostró la presencia de flavonoides, cumarinas, taninos y heterosidos cianogenéticos en las hojas de Cordia stellifera boraginaceae.

\section{METODOLOGÍA}

Diseño

Estudio descriptivo y transversal para determinar la presencia/ausencia de metabolitos secundario presentes en Cordia stellifera, que justifiquen el uso tradicional para combatir los efectos producidos por las mordeduras de serpientes.

\section{Población}

La selección de la planta en estudio se realizó a través del sondeo etnobotánico. La metodología de recolección de información se basó en entrevistas personales abiertas a miembros de diferentes edades de la comunidad de Lancetilla, usando como guía un cuestionario estructurado. La información recolectada incluye nombre común, parte de la planta utilizada, método de preparación y usos de diferentes plantas medicinales usadas en la región (ver figura 1). La selección de la comunidad se basó en el interés de los autores por aumentar el conocimiento científico sobre la flora de su comunidad de origen.

Se seleccionó el taxón Cordia stellifera boraginaceae como especie de estudio tomando como criterio la ausencia de reportes fitoquímicos y la relevancia del uso etnobotánico reportado para dicha especie.

\section{Entorno}

El sondeo etnobotánico se llevó a cabo en el mes de octubre de 2013, en la comunidad de Lancetilla, municipio de Tela, departamento de Atlántida. La recolección del material vegetal y el montaje de la muestra de herbario se realizaron en la comunidad de Lancetilla (ver figura 2). 


\section{Intervenciones}

Para realizar el análisis fitoquímico se desarrolló la metodología utilizada en el Laboratorio de farmacognosia I, Departamento de Control Químico, Facultad de Química y Farmacia, que se describe a continuación (Cáceres, 2003):

1. Material vegetal: la identificación taxonómica se realizó en el herbario Cyril Hardy Nelson Sutherland T, por la Dra. Lilian Ferrufino. Se depositó un ejemplar de respaldo en el herbario de la Facultad de Ciencias Químicas y Farmacia de la UNAH.

2. Procesamiento del material vegetal: las hojas se sometieron a un proceso de secado al aire bajo sombra a temperatura ambiente, posteriormente se pulverizaron utilizando un molino manual.

3. Obtención del extracto etanólico: $100 \mathrm{~g}$ de hojas secas y fragmentadas fueron sometidos a maceración dinámica por 3 horas y maceración estática por 7 días, en un recipiente color ámbar, utilizando como solvente de extracción etanol al $96 \%$ en cantidad suficiente para cubrir el material vegetal. Posteriormente, se clarificó por filtración al vacío y concentró mediante evaporación en plancha; se procedió a realizar las pruebas para cada uno de los metabolitos secundarios.

4. Se determinó la presencia/ ausencia de los siguientes núcleos:

a. Flavonoides: al extracto etanólico se realizaron pruebas con los reactivos: cloruro férrico, nitrato de plata, cloruro de aluminio y vapores de amoniaco; prueba de shinoda, álcalis, ácido sulfúrico, ácido sulfúrico y ácido bórico.

b. Cumarinas: 10 mililitros del extracto etanólico se colocan en un beaker que se sometió a una evaporación total en plancha, cubriendo el beaker con papel filtro tratado previamente con hidróxido de sodio al $10 \%$. Cuando se obtiene el extracto seco, se identifica la presencia de cumarinas en el papel filtro, utilizando luz ultravioleta para observar la fluorescencia verde azulada característica de estos compuestos.

c. Taninos: se pesan 10 gramos de droga seca y se adicionan 100 mililitros de agua destilada, se lleva a evaporación hasta obtener 10 mililitros, se filtra y se adiciona cloruro ferrico $1 \%$. Se observa un precipitado azul-negro en caso de ser positivo. 
d. Antraquinonas: pesar 10 gramos de muestra en un crisol, calcinar la muestra para favorecer la sublimación de las antraquinonas, el sublimado se recolecta en benceno y se le adiciona hidróxido de sodio al $10 \%$, observándose un precipitado color rojo si la prueba es positiva.

e. Glicósidos cardiotónicos: realizan las pruebas baljet, libermann- bauchatd, salkowski para determinar la presencia de estos heterósidos.

f. Heterósidos cianogeneticos: se pesan 10 gramos de material vegetal seco y se colocan en un beaker, se adiciona agua destilada hasta cubrir la muestra y se tapa con papel filtro previamente tratado con el reactivo de Grignard.

g. Alcaloides: se realizó una extracción hidroácida de las hojas, la presencia/ausencia de alcaloides se realiza mediante pruebas de precipitado con los reactivos mayer, dragendorff, wagner, hager, silicotungstico.

\section{RESULTADOS}

Los resultados del sondeo etnobotánico realizado a 30 personas de la comunidad de Lancetilla demostraron que la especie conocida comúnmente como sombra de ternero, es utilizada para tratar mordeduras de serpientes, lo que motivó el desarrollo del presente estudio químico de la especie y se desarrolló un protocolo de análisis fitoquímico. Los resultados de la determinación de grupos de metabolitos secundarios se muestran en el cuadro 1.

Cuadro 1. Análisis fitoquímico de Cordia stellifera boraginaceae

\section{Grupo químico: flavonoides}

\begin{tabular}{l|c|}
\hline \multicolumn{1}{|c|}{ Prueba } & Presencia/ausencia \\
\hline Shinoda & + \\
\hline Álcalis & + \\
Ácido sulfúrico & + \\
Ácido sulfúrico y ácido bórico & + \\
Cloruro férrico & + \\
Nitrato de plata & + \\
Cloruro de aluminio & - \\
Vapores de amoniaco & -
\end{tabular}




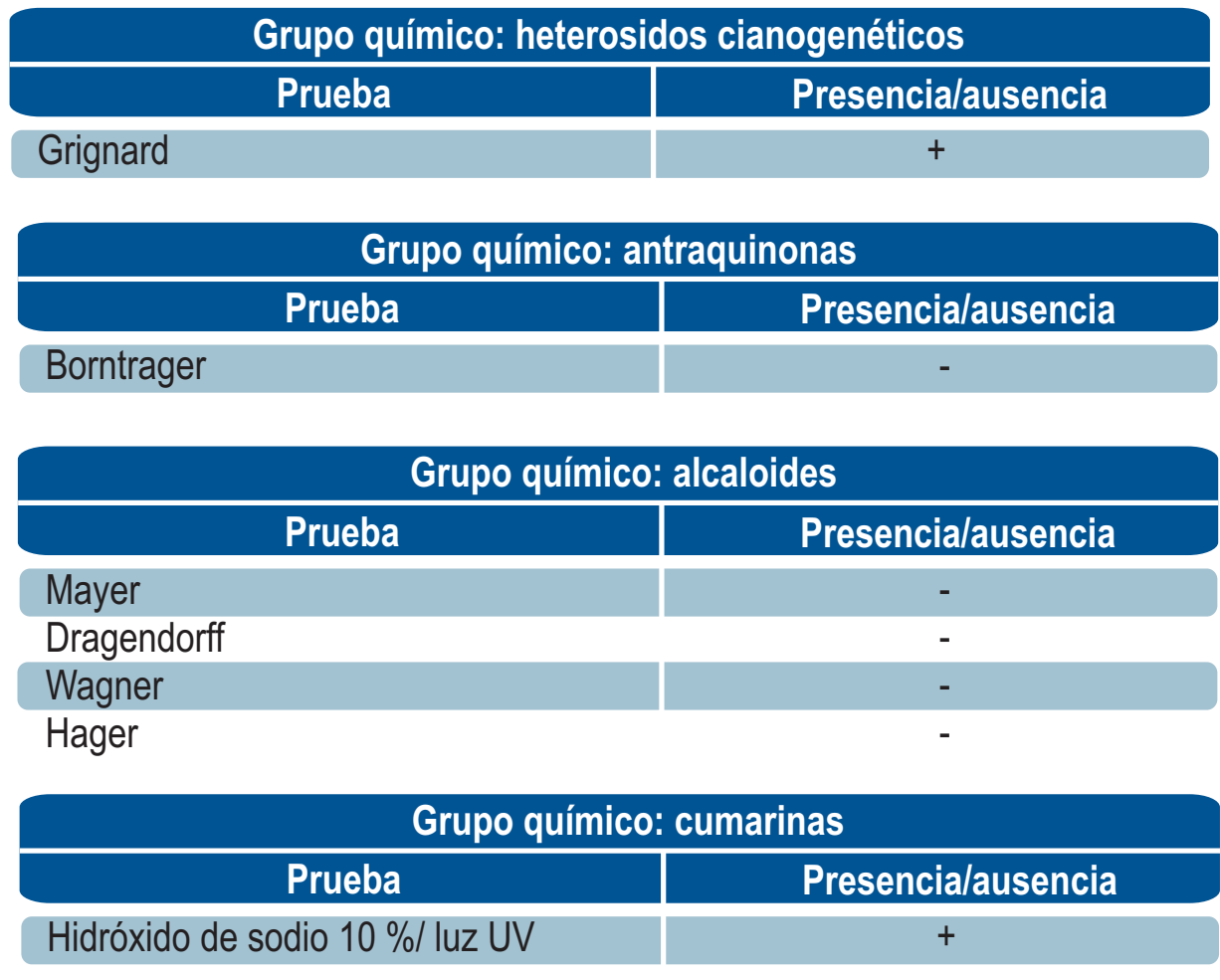

\begin{tabular}{|l|c|}
\hline \multicolumn{2}{|c|}{ Grupo químico: taninos } \\
\hline \multicolumn{1}{|c|}{ Prueba } & Presencia/ausencia \\
\hline Cloruro férrico $1 \%$ & + \\
\hline
\end{tabular}

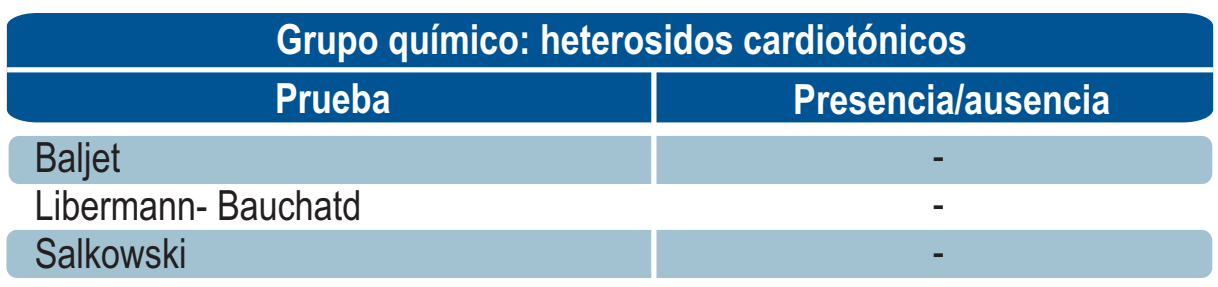

Significado de las claves: - ausencia, + presencia

\section{DISCUSIÓN}

En el presente análisis fitoquímico exploratorio de hojas de C. stellifera se partió de extractos etanólicos, puesto que este solvente tiene la capacidad de extraer compuestos de una amplia gama de polaridades, además de ser menos costoso y tóxico que otros solventes orgánicos. 
Esto permitió comprobar la presencia de flavonoides en la especie, pues en la literatura se reporta que algunos flavonoides son efectivos contra el veneno de los crotálidos; asímismo, se determinó la presencia taninos en las hojas, se sabe que ciertos taninos presentan efecto detoxificante y actividad contra ciertas serpientes marinas. La actividad de estos compuestos parece deberse a su capacidad de formar complejos con proteínas de venenos ofídicos, de ahí que plantas ricas en taninos como ciertas especies de acacia hayan sido tradicionalmente utilizados como antivenenosos en el área caribeña (Antonio, 2009).

También se identificó la presencia de cumarinas, metabolitos secundarios encontrados en algunas plantas con conocida actividad antiofídica. Registros en la literatura demuestran que las cumarinas, compuestos derivados de la ruta del ácido shikímico, son metabolitos muy activos biológicamente, cuya actividad antiofídica ya ha sido reportada previamente (Alarcon, 2008).

La familia de heterósidos cianogenéticos identificados en la planta tiene propiedades analgésicas, antiespasmódicas e hipotensoras.

\section{CONCLUSIONES}

De este estudio fitoquímico, se puede concluir que la especie C. stellifera produce flavonoides, cumarinas y taninos, que son compuestos que pueden ser responsables de la actividad antiofídica reportada por la comunidad de Lancetilla para esta la planta. Sin embargo, con los resultados de este estudio no se puede afirmar que la especie $C$. stellifera sea un antídoto contra las mordeduras de serpiente. Para evaluar la actividad antiofídica se recomienda realizar ensayos in vivo e in vitro de este taxón, además se sugiere continuar con el análisis fitoquímico para extraer, purificar y elucidar los compuestos presentes en la especie. Igualmente, se aconseja realizar las investigaciones con material en fresco de la planta recién colectada y compararlos con los resultados obtenidos con la planta seca.

\section{AGRADECIMIENTOS}

A todas las personas entrevistadas de la comunidad de Lancetilla, quienes tuvieron la bondad de brindarnos su tiempo y aportarnos sus valiosos conocimientos. 


\section{BIBLIOGRAFÍA}

Akerele, O. (1993). Las plantas medicinales, un tesoro que no debemos desperdiciar. Foro Mundial de la Salud. Revista Internacional de Desarrollo Sanitario, 14, 390-395. Ginebra

Alarcón P.; Martínez, J.C; Quintana, D.M.; Jiménez, J.C.; Díaz , S; Jiménez, I. (2008).Propagación in vitro de Renealmia alpinia (rottb), planta con actividad antiofídica. VITAE, Revista de la Facultad de Química Farmacéutica, Universidad de Antioquilla. 15(1), 61-69. Colombia.

Antonio, J.; Sáez, L.; Soto, J. P. (2009). Plantas alexitéricas: antídotos vegetales contra las picaduras de serpientes venenosas. Revista de Medicina Naturista 3(5), 17-24. España.

Beyra, Á.; León, M.; Iglesias, E. (2004). Estudios etnobotánicos sobre plantas medicinales en la provincia de Camagüey (Cuba). Anales del Jardín Botánico de Madrid, 61(2),185-204. España.

Cáceres, A.; Gattuso, S.; Guerrero, N.; Solís, P. (2003). Manual de caracterización y análisis de drogas vegetales y productos fitoquímicos. OEA. Guatemala.

David, J. Newman y Gordon, R. Cragg. (2012). Natural products as sources of new drugs over the 30 years from 1981 to 2010. Journal of Natural Products, 75, 311335. Estados Unidos de América.

De Menezes, J. E. S.; Machado, F. E.; Lemos, T. L. G.; Silveira, E. R.; Braz Filho, R. y Pessoa, O. D. L. (2004). Sesquiterpenes and a phenylpropanoid from Cordia trichotoma. Zeitschrift Für Naturforschung. C, Journal of Biosciences, 59(1-2), 19-22. India.

Nelson, Ciryl. (1986). Plantas comunes de Honduras.Tegucigalpa: Editorial Universitaria.

Paiva, R.; Lemos, T. L. G.; Pessoa, O. D. L.; Braz-filho, R.; Rodrigues-filho, E.; Viana, F. A. y Silveira, E. R. (2005). Chemical Constituents of Cordia piauhiensis - Boraginaceae. Journal of the Brazilian Chemical Society, 16(3), 662-665. Brasil.

Thirupathi, K.; Kumar, S. S.; Raju, V. S.; Ravikumar, B.; Krishna, D. R. y Mohan, G. K. (2008). A review of medicinal plants of the genus Cordia: Their chemistry and pharmacological uses. Journal of Natural Remedies, 8(1), 1-10. India. 
Figura 1. Encuestas etnobotánicas en la comunidad de Lancetilla, Atlántida

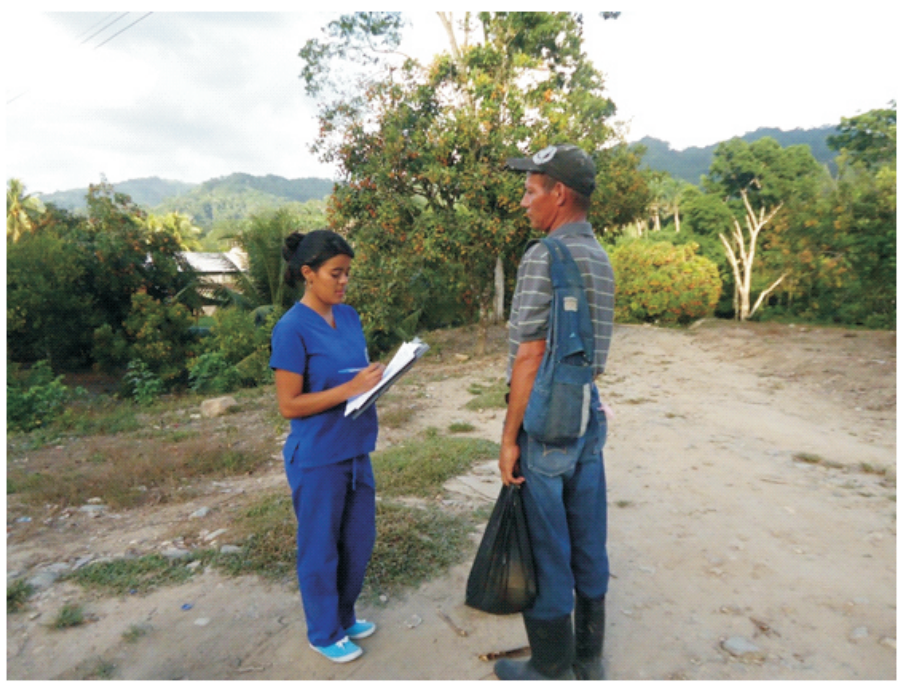

Figura 2. Recolección del material vegetal

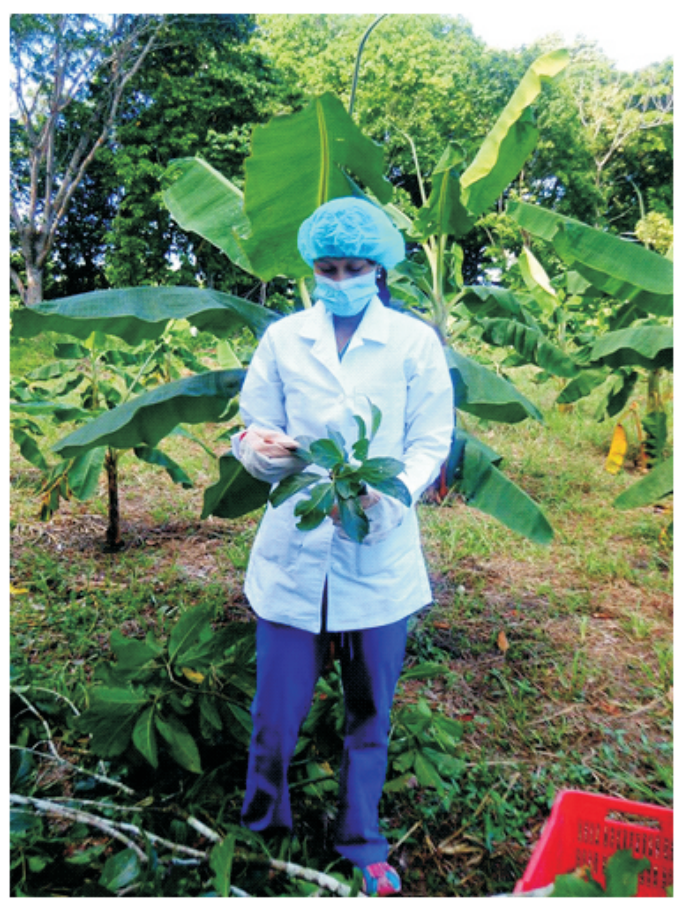

